Algebraic $\& \mathcal{G}$ Geometric $\mathcal{T}_{\text {opology }}$

Volume 1 (2001) 57-71

Published: 27 January 2001

ATG

\title{
On asymptotic dimension of groups
}

\author{
G. Bell and A. Dranishnikov
}

\begin{abstract}
We prove a version of the countable union theorem for asymptotic dimension and we apply it to groups acting on asymptotically finite dimensional metric spaces. As a consequence we obtain the following finite dimensionality theorems.

A) An amalgamated product of asymptotically finite dimensional groups has finite asymptotic dimension: $\operatorname{asdim} A *_{C} B<\infty$.

B) Suppose that $G^{\prime}$ is an HNN extension of a group $G$ with asdim $G<$ $\infty$. Then $\operatorname{asdim} G^{\prime}<\infty$.

C) Suppose that $\Gamma$ is Davis' group constructed from a group $\pi$ with asdim $\pi<\infty$. Then asdim $\Gamma<\infty$.
\end{abstract}

AMS Classification 20H15; 20E34, 20F69

Keywords Asymptotic dimension, amalgamated product, HNN extension

\section{Introduction}

The notion of the asymptotic dimension was introduced by Gromov [8] as an asymptotic analog of Ostrand's characterization of covering dimension. Two sets $U_{1}, U_{2}$ in a metric space are called $d$-disjoint if they are at least $d$-apart, i.e. $\inf \left\{\operatorname{dist}\left(x_{1}, x_{2}\right) \mid x_{1} \in U_{1}, x_{2} \in U_{2}\right\} \geq d$. A metric space $X$ has asymptotic dimension asdim $X \leq n$ if for an arbitrarily large number $d$ one can find $n+1$ uniformly bounded families $\mathcal{U}^{0}, \ldots, \mathcal{U}^{n}$ of $d$-disjoint sets in $X$ such that the union $\cup_{i} \mathcal{U}^{i}$ is a cover of $X$. A generating set $S$ in a group $\Gamma$ defines the word metric on $\Gamma$ by the following rule: $d_{S}(x, y)$ is the minimal length of a presentation of the element $x^{-1} y \in \Gamma$ in the alphabet $S$. Gromov applied the notion of asymptotic dimension to studying asymptotic invariants of discrete groups. It follows from the definition that the asymptotic dimension $\operatorname{asdim}\left(\Gamma, d_{S}\right)$ of a finitely generated group does not depend on the choice of the finite generating set $S$. Thus, asdim $\Gamma$ is an asymptotic invariant for finitely generated groups. Gromov proved [8] that asdim $\Gamma<\infty$ for hyperbolic groups $\Gamma$. The corresponding question about nonpositively curved (or CAT(0)) groups remains open. In the case of Coxeter groups it was answered in [7]. 
In [13] G. Yu proved a series of conjectures, including the famous Novikov Higher Signature conjecture, for groups $\Gamma$ with $\operatorname{asdim} \Gamma<\infty$. Thus, the problem of determining the asymptotic finite dimensionality of certain discrete groups became very important. In fact, until the recent example of Gromov [9] it was unknown whether all finitely presented groups satisfy the inequality asdim $\Gamma<$ $\infty$. In view of this, it is natural to ask whether the property of asymptotic finite dimensionality is preserved under the standard constructions with groups. Clearly, the answer is positive for the direct product of two groups. It is less clear, but still is not difficult to see that a semidirect product of asymptotically finite dimensional groups has a finite asymptotic dimension. The same question about the free product does not seem clear at all. In this paper we show that the asymptotic finite dimensionality is preserved by the free product, by the amalgamated free product and by the HNN extension.

One of the motivations for this paper was to prove that Davis' construction preserves asymptotic finite dimensionality. Given a group $\pi$ with a finite classifying space $B \pi$, Davis found a canonical construction, based on Coxeter groups, of a group $\Gamma$ with $B \Gamma$ a closed manifold such that $\pi$ is a retract of $\Gamma$ (see $[1],[2],[3],[10])$. We prove here that if $\operatorname{asdim} \pi<\infty$, then $\operatorname{asdim} \Gamma<\infty$. This theorem together with the result of the second author [6] (see also [5]) about the hypereuclideanness of asymptotically finite dimensional manifolds allows one to get a shorter and more elementary proof of the Novikov Conjecture for groups $\Gamma$ with $\operatorname{asdim} \Gamma<\infty$.

We note that the asymptotic dimension asdim is a coarse invariant, i.e. it is an invariant of the coarse category introduced in [11]. We recall that the objects in the coarse category are metric spaces and morphisms are coarsely proper and coarsely uniform (not necessarily continuous) maps. A map $f: X \rightarrow Y$ between metric spaces is called coarsely proper if the preimage $f^{-1}\left(B_{r}(y)\right)$ of every ball in $Y$ is a bounded set in $X$. A map $f: X \rightarrow Y$ is called coarsely uniform if there is a function $\rho: \mathbf{R}_{+} \rightarrow \mathbf{R}_{+}$, tending to infinity, such that $d_{Y}(f(x), f(y)) \leq \rho(d(x, y))$ for all $x, y \in Y$. We note that every object in the coarse category is isomorphic to a discrete metric space.

There is an analogy between the standard (local) topology and the asymptotic topology which is outlined in [4]. That analogy is not always direct. Thus, in Section 2 we prove the following finite union theorem for asymptotic dimension asdim $X \cup Y \leq \max \{$ asdim $X$, asdim $Y\}$ whereas the classical Menger-Urysohn theorem states: $\operatorname{dim} X \cup Y \leq \operatorname{dim} X+\operatorname{dim} Y+1$. Also the Countable Union Theorem in the classical dimension theory cannot have a straightforward analog, since all interesting objects in the coarse category are countable unions of points but not all of them are asymptotically 0-dimensional. In Section 2 we formulated a countable union theorem for asymptotic dimension which we 
found useful for applications to the case of discrete groups.

The second author was partially supported by NSF grant DMS-9971709.

\section{Countable union theorem}

Definition A family of metric spaces $\left\{F_{\alpha}\right\}$ satisfies the inequality asdim $F_{\alpha} \leq$ $n$ uniformly if for arbitrarily large $d>0$ there are $R$ and $R$-bounded $d$-disjoint families $\mathcal{U}_{\alpha}^{0} \ldots \mathcal{U}_{\alpha}^{n}$ of subsets of $F_{\alpha}$ such that the union $\cup_{i} \mathcal{U}_{\alpha}^{i}$ is a cover of $F_{\alpha}$.

A typical example of such family is when all $F_{\alpha}$ are isometric to a space $F$ with $\operatorname{asdim} F \leq n$.

A discrete metric space $X$ has bounded geometry if for every $R$ there is a constant $c=c(R)$ such that every $R$-ball $B_{R}(x)$ in $X$ contains at most $c$ points.

Proposition 1 Let $f_{\alpha}: F_{\alpha} \rightarrow X$ be a family of 1-Lipschitz injective maps to a discrete metric space of bounded geometry with asdim $X \leq n$. Then asdim $F_{\alpha} \leq n$ uniformly.

Proof For a metric space $A$ we define its $d$-components as the classes under the following equivalence relation. Two points $a, a^{\prime} \in A$ are equivalent if there is a chain of points $a_{0}, a_{1}, \ldots, a_{k}$ with $a_{0}=a, a_{k}=a^{\prime}$ and with $d\left(a_{i}, a_{i+1}\right) \leq d$ for all $i<k$. We note that the $d$-components are more than $d$ apart and also note that the diameter of each $d$-component is less than or equal to $d|A|$, where $|A|$ is the number of points in $A$.

Let $d$ be given. Then there are $R$-bounded $d$-disjoint families $\mathcal{V}^{0}, \ldots, \mathcal{V}^{n}$ covering $X$. For every $V \in \mathcal{V}^{i}$ and every $\alpha$ we present the set $f_{\alpha}^{-1}(V)$ as the union of $d$-components: $f_{\alpha}^{-1}(V)=\cup C_{\alpha}^{j}(V)$. Note that the diameter of every $d$-component is $\leq d c(R)$ where the function $c$ is taken from the bounded geometry condition on $X$. We take $\mathcal{U}_{\alpha}^{i}=\left\{C_{\alpha}^{j}(V) \mid V \in \mathcal{V}^{i}\right\}$.

Theorem 1 Assume that $X=\cup_{\alpha} F_{\alpha}$ and asdim $F_{\alpha} \leq n$ uniformly. Suppose that for any $r$ there exists $Y_{r} \subset X$ with asdim $Y_{r} \leq n$ and such that the family $\left\{F_{\alpha} \backslash Y_{r}\right\}$ is $r$-disjoint. Then asdim $X \leq n$.

Finite Union Theorem Suppose that a metric space is presented as a union $A \cup B$ of subspaces. Then asdim $A \cup B \leq \max \{$ asdim $A$, asdim $B\}$. 
Proof We apply Theorem 1 to the case when the family of subsets consists of $A$ and $B$ and we take $Y_{r}=B$.

The proof of Theorem 1 is based on the idea of saturation of one family by the other. Let $\mathcal{V}$ and $\mathcal{U}$ be two families of subsets of a metric space $X$.

Definition For $V \in \mathcal{V}$ and $d>0$ we denote by $N_{d}(V ; \mathcal{U})$ the union of $V$ and all elements $U \in \mathcal{U}$ with $d(V, U)=\min \{d(x, y) \mid x \in V, y \in U\} \leq d$. By $d$ saturated union of $\mathcal{V}$ and $\mathcal{U}$ we mean the following family $\mathcal{V} \cup_{d} \mathcal{U}=\left\{N_{d}(V ; \mathcal{U}) \mid\right.$ $V \in \mathcal{V}\} \cup\{U \in \mathcal{U} \mid d(U, V)>d$ for all $V \in \mathcal{V}\}$.

Note that this is not a commutative operation. Also note that $\{\emptyset\} \cup_{d} \mathcal{U}=\mathcal{U}$ and $\mathcal{V} \cup_{d}\{\emptyset\}=\mathcal{V}$ for all $d$.

Proposition 2 Assume that $\mathcal{U}$ is $d$-disjoint and $R$-bounded, $R \geq d$. Assume that $\mathcal{V}$ is $5 R$-disjoint and $D$-bounded. Then $\mathcal{V} \cup_{d} \mathcal{U}$ is d-disjoint and $D+$ $2(d+R)$-bounded.

Proof First we note that elements of type $U$ are $d$-disjoint in the saturated union. The same is true for elements of type $U$ and $N_{d}(V ; \mathcal{U})$. Now consider elements $N_{d}(V ; \mathcal{U})$ and $N_{d}\left(V^{\prime} ; \mathcal{U}\right)$. Note that they are contained in the $d+R$ neighborhoods of $V$ and $V^{\prime}$ respectively. Since $V$ and $V^{\prime}$ are $5 R$-disjoint, and $R \geq d$, the neighborhoods will be $d$-disjoint.

Clearly, $\operatorname{diam} N_{d}(V ; \mathcal{U}) \leq \operatorname{diam} V+2(d+R) \leq D+2(d+R)$.

Proof of Theorem 1 Let $d$ be given. Consider $R$ and families $\mathcal{U}_{\alpha}^{0} \ldots \mathcal{U}_{\alpha}^{n}$ from the definition of the uniform inequality asdim $F_{\alpha} \leq n$. We may assume that $R>d$. We take $r=5 R$ and consider $Y_{r}$ satisfying the conditions of the Theorem. Consider $r$-disjoint $D$-bounded families $\mathcal{V}^{0}, \ldots, \mathcal{V}^{k}$ from the definition of $\operatorname{asdim} Y_{r} \leq k$. Let $\overline{\mathcal{U}}_{\alpha}^{i}$ be the restriction of $\mathcal{U}_{\alpha}^{i}$ over $F_{\alpha} \backslash Y_{r}$, i.e. $\overline{\mathcal{U}}_{\alpha}^{i}=\left\{U \backslash Y_{r} \mid U \in \mathcal{U}_{\alpha}^{i}\right\}$. Let $\overline{\mathcal{U}}^{i}=\cup_{\alpha} \overline{\mathcal{U}}_{\alpha}^{i}$. Note that the family $\overline{\mathcal{U}}^{i}$ is $d$-disjoint and $R$-bounded. For every $i$ we define $\mathcal{W}^{i}=\mathcal{V}^{i} \cup_{d} \overline{\mathcal{U}}^{i}$. By Proposition 2 the family $\mathcal{W}^{i}$ is $d$-disjoint and uniformly bounded. Clearly $\cup_{i} \mathcal{W}^{i}$ covers $X$.

\section{Groups acting on finite dimensional spaces}

A norm on a group $A$ is a map \|\|$: A \rightarrow \mathbf{Z}_{+}$such that $\|a b\| \leq\|a\|+\|b\|$ and $\|x\|=0$ if and only if $x$ is the unit in $A$. A set of generators $S \subset A$ defines the norm $\|x\|_{S}$ as the minimal length of a presentation of $x$ in terms of $S$. A norm on a group defines a left invariant metric $d$ by $d(x, y)=\left\|x^{-1} y\right\|$. If $G$ 
is a finitely generated group and $S$ and $S^{\prime}$ are two finite generating sets, then the corresponding metrics $d_{S}$ and $d_{S^{\prime}}$ define coarsely equivalent metric spaces $\left(G, d_{S}\right)$ and $\left(G, d_{S^{\prime}}\right)$. In particular, $\operatorname{asdim}\left(G, d_{S}\right)=\operatorname{asdim}\left(G, d_{S^{\prime}}\right)$, and we can speak about the asymptotic dimension $\operatorname{asdim} G$ of a finitely generated group $G$.

Assume that a group $\Gamma$ acts on a metric space $X$. For every $R>0$ we define the $R$-stabilizer $W_{R}\left(x_{0}\right)$ of a point $x_{0} \in X$ as the set of all $g \in \Gamma$ with $g\left(x_{0}\right) \in B_{R}\left(x_{0}\right)$. Here $B_{R}(x)$ denotes the closed ball of radius $R$ centered at $x$.

Theorem 2 Assume that a finitely generated group $\Gamma$ acts by isometries on a metric space $X$ with a base point $x_{0}$ and with asdim $X \leq k$. Suppose that asdim $W_{R}\left(x_{0}\right) \leq n$ for all $R$. Then $\operatorname{asdim} \Gamma \leq(n+1)(k+1)-1$.

Proof We define a map $\pi: \Gamma \rightarrow X$ by the formula $\pi(g)=g\left(x_{0}\right)$. Then $W_{R}\left(x_{0}\right)=\pi^{-1}\left(B_{r}\left(x_{0}\right)\right)$. Let $\lambda=\max \left\{d_{X}\left(s\left(x_{0}\right), x_{0}\right) \mid s \in S\right\}$. We show now that $\pi$ is $\lambda$-Lipschitz. Since the metric $d_{S}$ on $\gamma$ is induced from the geodesic metric on the Cayley graph, it suffices to check that $d_{X}\left(\pi(g), \pi\left(g^{\prime}\right)\right) \leq \lambda$ for all $g, g^{\prime} \in \Gamma$ with $d_{S}\left(g, g^{\prime}\right)=1$. Without loss of generality we assume that $g^{\prime}=g s$ where $s \in S$. Then $d_{X}\left(\pi(g), \pi\left(g^{\prime}\right)\right)=d_{X}\left(g\left(x_{0}\right), g s\left(x_{0}\right)\right)=d_{X}\left(x_{0}, s\left(x_{0}\right)\right) \leq \lambda$.

Note that $\gamma B_{R}(x)=B_{R}(\gamma(x))$ and $\gamma\left(\pi^{-1}\left(B_{R}(x)\right)\right)=\pi^{-1}\left(B_{R}(\gamma(x))\right)$ for all $\gamma \in \Gamma, x \in X$ and all $R$.

Given $r>0$, there are $\lambda r$-disjoint, $R$-bounded families $\mathcal{F}^{0}, \ldots, \mathcal{F}^{k}$ on the orbit $\Gamma x_{0}$. Let $\mathcal{V}^{0}, \ldots, \mathcal{V}^{n}$ on $W_{2 R}\left(x_{0}\right)$ be $r$-disjoint uniformly bounded families given by the definition of the inequality $\operatorname{asdim} W_{R}\left(x_{0}\right) \leq n$. For every element $F \in \mathcal{F}^{i}$ we choose an element $g_{F} \in \Gamma$ such that $g_{F}\left(x_{0}\right) \in F$. We define $(k+1)(n+1)$ families of subsets of $\Gamma$ as follows:

$$
\mathcal{W}^{i j}=\left\{g_{F}(C) \cap \pi^{-1}(F) \mid F \in \mathcal{F}^{i}, C \in \mathcal{V}^{j}\right\}
$$

Since multiplication by $g_{F}$ from the left is an isometry, every two distinct sets $g_{F}(C)$ and $g_{F}\left(C^{\prime}\right)$ are $r$-disjoint. Note that $\pi\left(g_{F}(C) \cap \pi^{-1}(F)\right)$ and $\pi\left(g_{F^{\prime}}(C) \cap \pi^{-1}\left(F^{\prime}\right)\right)$ are $\lambda r$-disjoint for $F \neq F^{\prime}$. Since $\pi$ is $\lambda$-Lipschitz, the sets $g_{F}(C) \cap \pi^{-1}(F)$ and $g_{F^{\prime}}\left(C^{\prime}\right) \cap \pi^{-1}\left(F^{\prime}\right)$ are $r$-disjoint. The families $\mathcal{W}^{i j}$ are uniformly bounded, since the families $\mathcal{V}^{j}$ are, and multiplication by $g$ from the left is an isometry on $\Gamma$. We check that the union of the families $\mathcal{W}^{i j}$ forms a cover of $\Gamma$. Let $g \in \Gamma$ and let $\pi(g)=F$, i.e. $g\left(x_{0}\right) \in F$. Since $\operatorname{diam} F \leq R$, $x_{0} \in g_{F}^{-1}(F) \leq R$ and $g_{F}^{-1}$ acts as an isometry, we have $g_{F}^{-1}(F) \subset B_{R}\left(x_{0}\right)$. Therefore, $g_{F}^{-1} g\left(x_{0}\right) \in B_{R}\left(x_{0}\right)$, i. e. $g_{F}^{-1} g \in W_{R}\left(x_{0}\right)$. Hence $g_{F}^{-1} g$ lies in some set $C \in \mathcal{V}^{j}$ for some $j$. Therefore $g \in g_{F}(C)$. Thus, $g \in g_{F}(C) \cap \pi^{-1}(F)$. 
Theorem 3 Let $\phi: G \rightarrow H$ be an epimorphism of a finitely generated group $G$ with kernel $\operatorname{ker} \phi=K$. Assume that asdim $K \leq k$ and asdim $H \leq n$. Then $\operatorname{asdim} G \leq(n+1)(k+1)-1$.

Proof The group $G$ acts on $H$ by the rule $g(h)=\phi(g) h$. This is an action by isometries for every left invariant metric on $H$. Let $S$ be a finite generating set for $G$. We consider the metric on $H$ defined by the set $\phi(S)$. Below we prove that the $R$-stabilizer of the identity $W_{R}(e)$ coincides with $N_{R}(K)$, the $R$-neighborhood of $K$ in $G$. Since $N_{R}(K)$ is coarsely isomorphic to $K$, we have the inequality $\operatorname{asdim} W_{R}(e) \leq k$.

Let $g \in W_{R}(e)$, then $\|\phi(g)\| \leq R$. Therefore, there is a sequence $i_{1}, \ldots, i_{k}$ with $k \leq R$ such that $\phi(g)=\bar{s}_{i_{1}} \ldots \bar{s}_{i_{k}}$ where $\bar{s}=\phi(s), s \in S$. Let $u=s_{i_{1}} \ldots s_{i_{k}}$. Then $d_{S}\left(g, g u^{-1}\right) \leq R$ and hence, $d_{S}(g, K) \leq R$. In the opposite direction, if $d_{S}(g, K) \leq R$, then $d(g, z) \leq R$ for some $z \in K$. Hence $d_{\phi(S)}(\phi(g), e) \leq R$.

We apply Theorem 2 to complete the proof.

Remark The estimate $(n+1)(k+1)-1$ in Theorems 2 and 3 is far from being sharp. Since in this paper we are interested in finite dimensionality only, we are not trying to give an exact estimate which is $n+k$. Besides, it would be difficult to get an exact estimate just working with covers. Even for proving the inequality

$$
\operatorname{asdim} \Gamma_{1} \times \Gamma_{2} \leq \operatorname{asdim} \Gamma_{1}+\operatorname{asdim} \Gamma_{2}
$$

it is better to use a different approach to asdim (see [7]).

\section{Free and amalgamated products}

Let $\left\{A_{i},\|\|_{i}\right\}$ be a sequence of groups with norms. Then these norms generate a norm on the free product $* A_{i}$ as follows. Let $x_{i_{1}} x_{i_{2}} \ldots x_{i_{m}}$ be the reduced presentation of $x \in * A_{i}$, where $x_{i_{k}} \in A_{i_{k}}$. We denote by $l(x)=m$ the length of the reduced presentation of $x$ and we define $\|x\|=\left\|x_{i_{1}}\right\|_{i_{1}}+\ldots+\left\|x_{i_{m}}\right\|_{i_{m}}$.

Theorem 4 Let $\left\{A_{i},\|\|_{i}\right\}$ be a sequence of groups satisfying asdim $A_{i} \leq n$ uniformly and let \|\| be the norm on the free product $* A_{i}$ generated by the norms \|\|$_{i}$. Then $\operatorname{asdim}\left(* A_{i},\|\|\right) \leq 2 n+1$. 
Proof First we note that the uniform property $\operatorname{asdim} A_{i} \leq n$ and Theorem 1 applied with $Y_{r}=B_{r}(e)$, the $r$-ball in $* A_{i}$ centered at the unit $e$, imply that asdim $\cup A_{i} \leq n$.

We let $G$ denote $* A_{i}$. Then we consider a tree $T$ with vertices left cosets $x A_{j}$ in $G$. Two vertices $x A_{i}$ and $y A_{j}$ are joined by an edge if and only if there is an element $z \in G$ such that $x A_{i}=z A_{i}$ and $y A_{j}=z A_{j}$ and $i \neq j$. The multiplication by elements of $G$ from the left defines an action of $G$ on $T$. We note that the $m$-stabilizer $W_{m}\left(A_{1}\right)$ of the vertex $A_{1}$ is the union of all possible products $A_{i_{1}} \ldots A_{i_{l}} A_{1}$ of the length $\leq m+1$, where $i_{k} \neq i_{k+1}$ and $i_{l} \neq 1$. Let $P_{m}=\left\{x \in * A_{i} \mid l(x)=m\right\}$ and let $P_{m}^{k}=\left\{x \in P_{m} \mid x=x_{i_{1}} \ldots x_{i_{m}}, x_{i_{m}} \notin\right.$ $\left.A_{k}\right\}$. Put $R_{m}=W_{m}\left(A_{1}\right) \backslash W_{m-1}\left(A_{1}\right)$. Then $R_{m} \subset P_{m+1}$

By induction on $m$ we show that $\operatorname{asdim} P_{m} \leq n$. This statement holds true when $m=0$, since $P_{0}=\{e\}$. Assume that it holds for $P_{m-1}$. We note that $P_{m}=\cup_{x \in P_{m-1}^{i}} x A_{i}$. Since multiplication from the left is an isometry, the hypothesis of the theorem implies that the inequality asdimx $A_{i} \leq n$ holds uniformly. Given $r$ we consider the set $Y_{r}=P_{m-1} B_{r}(e)$ where $B_{r}(e)$ is the $r$-ball in $* A_{i}$. Since $Y_{r}$ contains $P_{m-1}$ and is contained in $r$-neighborhood of $P_{m-1}$, it is isomorphic in the coarse category to $P_{m-1}$. Hence by the induction assumption we have asdim $Y_{r} \leq n$. We show that the family $x A_{i} \backslash Y_{r}, x \in P_{m-1}^{i}$ is $r$-disjoint. Assume that $x A_{i} \neq x^{\prime} A_{j}$. This means that $x \neq x^{\prime}$ if $i=j$. If $i \neq j$ the inequality $\left\|a_{i}^{-1} x^{-1} x^{\prime} a_{j}\right\| \geq\left\|a_{i}^{-1} a_{j}\right\|=\left\|a_{i}\right\|+\left\|a_{j}\right\|$ holds for any choice of $a_{i} \in A_{i}$ and $a_{j} \in A_{j}$. If $i=j$, the same inequality holds, since $x \neq x^{\prime}$ and they are of the same length. If $x a_{i} \in x A_{i} \backslash Y_{r}$ and $x^{\prime} a_{j} \in x A_{j} \backslash Y_{r}$, then $\left\|a_{i}\right\|,\left\|a_{j}\right\| \geq r$ and hence $\operatorname{dist}\left(x a_{i}, x^{\prime} a_{j}\right) \geq 2 r$. Theorem 1 implies that $\operatorname{asdim}_{m} \leq n$. The Finite Union Theorem implies that $\operatorname{asdim} W_{m}\left(A_{1}\right) \leq n$ for all $n$.

It is known that every tree $T$ has asdim $T=1$ (see [7]). Thus by Theorem 2 $\operatorname{asdim}\left(* A_{i},\|\|\right) \leq 2 n+1$.

Corollary Let $A_{i}, i=1, \ldots, k$, be finitely generated groups with asdim $A_{i} \leq$ $n$. Then asdim $*_{i=1}^{k} A_{i} \leq 2 n+1$.

Theorem 5 Let $A$ and $B$ be finitely generated groups with asdim $A \leq n$ and asdim $B \leq n$ and let $C$ be their common subgroup. Then asdim $A *_{C} B \leq$ $2 n+1$.

We recall that every element $x \in A *_{C} B$ admits a unique normal presentation $c \bar{x}_{1} \ldots \bar{x}_{k}$ where $c \in C, \bar{x}_{i}=C x_{i}$ are nontrivial alternating right cosets of $C$ in $A$ or $B$. Thus, $x=c x_{1} \ldots x_{k}$. Let $\operatorname{dist}($,$) be a met-$ ric on the group $G=A *_{C} B$. We assume that this metric is generated 
by the union of the finite sets of generators $S=S_{A} \cup S_{B}$ of the groups $A$ and $B$. On the space of the right cosets $C \backslash G$ of a subgroup $C$ in $G$ one can define the metric $\bar{d}(C x, C y)=\operatorname{dist}(C x, C y)=\operatorname{dist}(x, C y)$. The following chain of inequalities implies the triangle inequality for $\bar{d}$ : $\operatorname{dist}(C a, C b) \leq$ $\operatorname{dist}\left(a, c^{\prime} b\right)=\left\|a^{-1} c^{\prime} b\right\| \leq\left\|a^{-1} c z\right\|+\left\|(c z)^{-1} c^{\prime} b\right\|=\operatorname{dist}(a, c z)+\operatorname{dist}\left(c z, c^{\prime} b\right)$. We chose $c$ such that $\operatorname{dist}(a, c z)=\operatorname{dist}(a, C z)=\bar{d}(C a, C z)$ and $c^{\prime}$ such that $\operatorname{dist}\left(c z, c^{\prime} b\right)=\operatorname{dist}(c z, C b)=\bar{d}(C z, C b)$.

For every pair of pointed metric spaces $X$ and $Y$ we define a free product $X \hat{*} Y$ as a metric space whose elements are alternating words formed by the alphabets $X \backslash\left\{x_{0}\right\}$ and $Y \backslash\left\{y_{0}\right\}$ plus the trivial word $x_{0}=y_{0}=\tilde{e}$. We define the norm of the trivial word to be zero and for a word of type $x_{1} y_{1} \ldots x_{r} y_{r}$ we set $\left\|x_{1} y_{1} \ldots x_{r} y_{r}\right\|=\Sigma_{i} d_{X}\left(x_{i}, x_{0}\right)+d_{Y}\left(y_{i}, y_{0}\right)$. If the word starts or ends by a different type of letter, we consider the corresponding sum. To define the distance $d\left(w, w^{\prime}\right)$ between two words $w$ and $w^{\prime}$ we cut off their common part $u$ if it is not empty: $w=u x v, w^{\prime}=u x^{\prime} v^{\prime}$ and set $d\left(w, w^{\prime}\right)=d\left(x, x^{\prime}\right)+\|v\|+\left\|v^{\prime}\right\|$. If the common part is empty, we define $d\left(w, w^{\prime}\right)=\|w\|+\left\|w^{\prime}\right\|$. Thus, $d(w, \tilde{e})=$ $\|w\|$.

Proposition 3 Let $c \bar{x}_{1} \ldots \bar{x}_{r}$ be the normal presentation of $x \in A *_{C} B$. Then $\|x\| \geq \Sigma_{i} \bar{d}\left(\bar{x}_{i}, C\right)$.

Proof We define a map $\phi: A *_{C} B \rightarrow(C \backslash A) \hat{*}(C \backslash B)$ as follows. If $c \bar{x}_{1} \ldots \bar{x}_{r}$ is the normal presentation of $x$, then we set $\phi(x)=\bar{x}_{1} \ldots \bar{x}_{r}$ and define $\phi(e)=\tilde{e}$. We verify that $\phi$ is 1-Lipschitz. Since $A *_{C} B$ is a discrete geodesic metric space space, it suffices to show that $d(\phi(x), \phi(x \gamma)) \leq 1$ where $\gamma$ is a generator in $A$ or in $B$. Let $x=c x_{1} \ldots x_{r}$ be a presentation corresponding to the normal presentation $c \bar{x}_{1} \ldots \bar{x}_{r}$. Then the normal presentation of $x \gamma$ will be either $c \bar{x}_{1} \ldots\left(\overline{x_{r} \gamma}\right)$ or $c \bar{x}_{1} \ldots \bar{x}_{r} \bar{\gamma}$. In the first case, $d(\phi(x), \phi(x \gamma))=$ $\bar{d}\left(\bar{x}_{r}, \overline{x_{r} \gamma}\right)=\operatorname{dist}\left(C x_{r}, C x_{r} \gamma\right) \leq \operatorname{dist}\left(x_{r}, x_{r} \gamma\right)=1$. In the second case we have $d(\phi(x), \phi(x \gamma))=\bar{d}(C, C \gamma)=\operatorname{dist}(C, C \gamma) \leq \operatorname{dist}(e, \gamma)=1$.

Then $\|x\|=\operatorname{dist}(x, e) \geq d(\phi(x), \tilde{e})=d\left(\bar{x}_{1} \ldots \bar{x}_{r}, \tilde{e}\right)=\left\|x_{1} \ldots x_{r}\right\|=\Sigma_{i} \bar{d}\left(\bar{x}_{i}, \bar{e}\right)$.

Proposition 4 Suppose that the subset $(B A)^{m}=B A \ldots B A \subset A *_{C} B$ is supplied with the induced metric and let asdim $A, \operatorname{asdim} B \leq n$. Then $\operatorname{asdim}(B A)^{m} \leq n$ for all $m$. 
Proof Let $l(x)$ denote the length of the normal presentation $c \bar{x}_{1} \ldots \bar{x}_{l(x)}$ of $x$. Define $P_{k}=\{x \mid l(x)=k\}, P_{k}^{A}=\left\{x \in P_{k} \mid x_{l(x)} \in C \backslash A\right\}$ and $P_{k}^{B}=\left\{x \in P_{k} \mid\right.$ $\left.x_{l(x)} \in C \backslash B\right\}$. Note that $P_{k}=P_{k}^{A} \cup P_{k}^{B}$. Also we note that $(B A)^{m} \subset \cup_{k=1}^{2 m} P_{k}$. In view of the Finite Union Theorem it is sufficient to show that asdim $P_{k} \leq n$ for all $k$. We proceed by induction on $k$. It is easy to see that $P_{k+1}^{A} \subset P_{k}^{B} A$. Assuming the inequality $\operatorname{asdim} P_{k} \leq n$, we show that $\operatorname{asdim} P_{k}^{B} A \leq n$. We define $Y_{r}=P_{k} N_{r}^{A}(C)$ where $N_{r}^{A}(C)$ denotes an $r$-neighborhood of $C$ in $A$. First we show that $Y_{r} \subset N_{r}\left(P_{k}\right)$. Let $y \in Y_{r}$, then $y$ has the form $u z$ where $u \in$ $P_{k}^{B}, z \in A$ and $\operatorname{dist}(z, C) \leq r$, i.e. $\left\|z^{-1} c\right\| \leq r$ for some $c \in C$. Let $c^{\prime} \bar{x}_{1} \ldots \bar{x}_{k}$ be the normal presentation of $u$, then $u z=c^{\prime} x_{1} x_{2} \ldots x_{k-1} x_{k} z$ where $x_{k} \in$ $B \backslash C$. We note that the element $u c$ has the normal presentation $c^{\prime} \bar{x}_{1} \ldots \overline{x_{k} c}$ and hence $u c \in P_{k}$. Then $\left.\operatorname{dist}(y, u c)\right)=\left\|z^{-1} c\right\| \leq r$, therefore $\operatorname{dist}\left(y, P_{k}\right) \leq r$, i.e. $y \in N_{r}\left(P_{k}\right)$. Since the $r$-neighborhood $N_{r}\left(P_{k}\right)$ is coarsely isomorphic to the space $P_{k}$, by the induction assumption we have $\operatorname{asdim} N_{r}\left(Y_{r}\right) \leq n$ and hence, $\operatorname{asdim} Y_{r} \leq n$.

We consider families $x A$ with $x \in P_{k}^{B}$. Let $x A$ and $x^{\prime} A$ be two different cosets. Since $x$ and $x^{\prime}$ are different elements with $l(x)=l\left(x^{\prime}\right)$, and $x^{-1} x^{\prime} \notin A$, the normal presentation of $a^{-1} x^{-1} x^{\prime} a^{\prime}$ ends by the coset $C a^{\prime}$.

Then by Proposition $3 \operatorname{dist}\left(x A \backslash Y_{r}, x^{\prime} A \backslash Y_{r}\right)=\left\|a^{-1} x^{-1} x^{\prime} a^{\prime}\right\| \geq \bar{d}\left(C a^{\prime}, C\right)=$ $\operatorname{dist}\left(C a^{\prime}, C\right)=\operatorname{dist}\left(a^{\prime}, C\right)>r$. Note that $P_{k}^{B} A$ is the union of these sets $x A$. Since all $x A$ are isometric, we have a uniform inequality asdim $x A \leq n$. According to Theorem 1 we obtain that $\operatorname{asdim} P_{k}^{B} A \leq n$ and hence $\operatorname{asdim} P_{k+1}^{A} \leq n$. Similarly one obtains the inequality $\operatorname{asdim} P_{k+1}^{B} \leq n$. The Finite Union Theorem implies that asdim $P_{k+1} \leq n$.

Proof of Theorem 5 We define a graph $T$ as follows. The vertices of $T$ are the left cosets $x A$ and $y B$. Two vertices $x A$ and $y B$ are joined by an edge if there is $z$ such that $x A=z A$ and $y B=z B$. To check that $T$ is a tree we introduce the weight of a vertex $Y \in T$ given by $w(Y)=\min \{l(y) \mid y \in Y\}$. Note that for every vertex $e$ with $w(e)>0$ there is a unique neighboring vertex $e_{-}$with $w\left(e_{-}\right)<w(e)$. Since we always have $w(z A) \neq w(z B)$, we get an orientation on $T$ with $w\left(e_{-}\right)<w\left(e_{+}\right)$for every edge $e$. The existence this orientation implies that $T$ does not contain cycles. Since every vertex of $T$ can be connected with the vertex $A$, the graph $T$ is connected. Thus, $T$ is a tree. The action of $A *_{C} B$ on $T$ is defined by left multiplication. We note that the $k$-stabilizer $W_{k}(A)$ is contained in $(B A)^{k}$. Then by Proposition 4 $\operatorname{asdim}_{k}(A) \leq n$. By Theorem $2 \operatorname{asdim} A *_{C} B \leq 2 n+1$.

Let $\left\{A_{i},\|\|_{i}\right\}$ be a sequence of groups with norms and let $C \subset A_{i}$ be a common subgroup. These norms define a norm \|\| on the amalgamated product ${ }^{*} C A_{i}$ 
by taking $\|x\|$ equal the minimum of sums $\Sigma_{k=1}^{l}\left\|a_{i_{k}}\right\|_{i_{k}}$ where $x=a_{i_{1}} \ldots a_{i_{l}}$ and $a_{i_{k}} \in A_{i_{k}}$.

The following theorem generalizes Theorem 4 and Theorem 5 .

Theorem 6 Let $\left\{A_{i},\|\|_{i}\right\}$ be a sequence of groups satisfying asdim $A_{i} \leq n$ uniformly and let \|\| be the norm on a free product $* A_{i}$ generated by the norms \|\|$_{i}$. Let $C$ be a common subgroup. Then $\operatorname{asdim}\left(*_{C} A_{i},\|\|\right) \leq 2 n+1$.

The proof is omitted since it follows exactly the same scheme.

The following fact will be used in Section 6 in the case of the free product.

Proposition 5 Assume that the groups $A_{i}$ are supplied with the norms which generate the norm on the amalgamated product ${ }^{*} C A_{i}$. Let $\psi:{ }_{C} A_{i} \rightarrow \Gamma$ be a monomorphism to a finitely generated group such that the restriction $\left.\psi\right|_{A_{i}}$ is an isometry for every $i$. Then $\psi$ is a coarsely uniform embedding.

Proof Since $\psi$ is a bijection onto the image, both maps $\psi$ and $\psi^{-1}$ are coarsely proper. We check that both are coarsely uniform. First we show that $\psi$ is 1-Lipschitz. Let $x, y \in{ }^{*} A_{i}$ and let $x^{-1} y=a_{i_{1}} \ldots a_{i_{n}}$ with $\left\|x^{-1} y\right\|=$ $\sum_{k=1}^{n}\left\|a_{i_{k}}\right\|_{i_{k}}$. Then $d_{\Gamma}(\psi(x), \psi(y)) \leq$

$d_{\Gamma}\left(\psi(x), \psi\left(x a_{i_{1}}\right)\right)+d_{\Gamma}\left(\psi\left(x a_{i_{1}}\right), \psi\left(x a_{i_{1}} a_{i_{2}}\right)\right)+\ldots+d_{\Gamma}\left(\psi\left(x a_{i_{1}} \ldots a_{i_{n-1}}\right), \psi(y)\right)$

$=\Sigma_{k=1}^{n}\left\|\psi\left(a_{i_{k}}\right)\right\|_{\Gamma}=\Sigma_{k=1}^{n}\left\|a_{i_{k}}\right\|_{i_{k}}=\left\|x^{-1} y\right\|=\operatorname{dist}(x, y)$.

Now we show that $\psi^{-1}$ is uniform. For every $r$ the preimage $\psi^{-1}\left(B_{r}(e)\right)$ is finite, since $B_{r}(e)$ is finite and $\psi$ is injective. We define $\xi(r)=\max \{\|z\| \mid z \in$ $\left.\psi^{-1}\left(B_{r}(e)\right)\right\}$. Let $\bar{\xi}$ be strictly monotonic function which tends to infinity and $\bar{\xi} \geq \xi$. Let $\rho$ be the inverse function of $\bar{\xi}$. Then

$$
\begin{aligned}
& d_{\Gamma}(\psi(x), \psi(y))=\left\|\psi\left(x^{-1} y\right)\right\|_{\Gamma}=\rho\left(\bar{\xi}\left(\left\|\psi\left(x^{-1} y\right)\right\|_{\Gamma}\right)\right) \geq \rho\left(\xi\left(\left\|\psi\left(x^{-1} y\right)\right\|_{\Gamma}\right)\right) \geq \\
& \rho\left(\left\|x^{-1} y\right\|\right)=\rho(d(x, y))
\end{aligned}
$$

The last inequality follows from the inequality $\xi(\|\psi(z)\|) \geq\|z\|$ and the fact that $\rho$ is an increasing function.

\section{$5 \quad$ HNN extension}

Let $A$ be a subgroup of a group $G$ and let $\phi: A \rightarrow G$ be a monomorphism. We denote by $G^{\prime}$ the HNN extension of $G$ by means of $\phi$, i.e. a group $G^{\prime}$ generated by $G$ and an element $y$ with the relations $y a y^{-1}=\phi(a)$ for all $a \in A$. 
Theorem 7 Let $\phi: A \rightarrow G$ be a monomorphism of a subgroup $A$ of a group $G$ with asdim $G \leq n$ and let $G^{\prime}$ be the HNN extension of $G$. Then $\operatorname{asdim} G^{\prime} \leq 2 n+1$.

We recall that a reduced presentation of an element $x \in G^{\prime}$ is a word

$$
g_{0} y^{\epsilon_{1}} g_{1} \ldots y^{\epsilon_{n}} g_{n}=x,
$$

where $g_{i} \in G, \epsilon_{i}= \pm 1$, with the property that $g_{i} \notin A$ whenever $\epsilon_{i}=1$ and $\epsilon_{i+1}=-1$ and $g_{i} \notin \phi(A)$ whenever $\epsilon_{i}=-1$ and $\epsilon_{i+1}=1$. The number $n$ is called the length of the reduced presentation $g_{0} y^{\epsilon_{1}} g_{1} \ldots y^{\epsilon_{n}} g_{n}$.

The following facts are well-known [12]:

A) (uniqueness) Every two reduced presentations of the same element have the same length and can be obtained from each other by a sequence of the following operations:

(1) replacement of $y$ by $\phi(a) y a^{-1}$,

(2) replacement of $y^{-1}$ by $a^{-1} y \phi(a), a \in A$

B) (existence) Every word of type $g_{0} y^{\epsilon_{1}} g_{1} \ldots y^{\epsilon_{n}} g_{n}$ can be deformed to a reduced form by a sequence of the following operations:

(1) replacement of $y g y^{-1}$ by $\phi(g)$ for $g \in A,(2)$ replacement of $y^{-1} \phi(g) y$ by $g$ for $g \in A$, (3) replacement of $g^{\prime} \bar{g}$ by $g=g^{\prime} \bar{g} \in G$ if $g^{\prime}, \bar{g} \in G$.

In particular the uniqueness implies that for any two reduced presentations $g_{0} y^{\epsilon_{1}} g_{1} \ldots y^{\epsilon_{n}} g_{n}$ and $g_{0}^{\prime} y^{\epsilon_{1}^{\prime}} g_{1} \ldots y^{\epsilon_{n}^{\prime}} g_{n}^{\prime}$ of the same element $x \in G^{\prime}$ we have $\left(\epsilon_{1}, \ldots, \epsilon_{n}\right)=\left(\epsilon_{1}^{\prime}, \ldots, \epsilon_{n}^{\prime}\right)$.

Let $G$ be a finitely generated group and let $S$ be a finite set of generators. We consider the norm on $G^{\prime}$ defined by the generating set $S^{\prime}=S \cup\left\{y, y^{-1}\right\}$.

Proposition 6 Let $g_{0} y^{\epsilon_{1}} g_{1} \ldots y^{\epsilon_{n}} g_{n}$ be a reduced presentation of $x \in G^{\prime}$. Then $\|x\| \geq d\left(g_{n}, A\right)$ if $\epsilon_{n}=1$ and $\|x\| \geq d\left(g_{n}, \phi(A)\right)$ if $\epsilon_{n}=-1$.

Proof We consider here the case when $\epsilon_{n}=1$. A shortest presentation of $x$ in the alphabet $S^{\prime}$ gives rise an alternating presentation $x=r_{0}^{0} \epsilon^{\epsilon_{1}^{0}} r_{1}^{0} \ldots y^{\epsilon_{m_{0}}^{0}} r_{m_{0}}^{0}$, $r_{i}^{0} \in G, \epsilon_{i}^{0}= \pm 1$ with $\|x\|=m_{0}+\left\|r_{0}^{0}\right\|+\ldots+\left\|r_{m_{0}}^{0}\right\|$. We consider a sequence of presentations of $x$ connecting the above presentation with a reduced presentation $r_{0}^{1} y^{\epsilon_{1}^{1}} r_{1}^{1} \ldots y^{\epsilon_{m_{1}}^{1}} r_{m_{1}}^{1}$ by means of operations (1)-(3) of B). Then by A) we have that $m_{1}=n, \epsilon_{n}^{1}=\epsilon_{n}=1$ and $g_{n}=\tilde{a} r_{n}^{1}, \tilde{a} \in A$. Because of the nature of transformations (1)-(3) of B), we can trace out to the shortest presentation the letter $y=y^{\epsilon_{n}^{1}}$ from the reduced word. This means that the 0 -th word has 
the form $r_{0}^{0} y^{\epsilon_{1}^{0}} r_{1}^{0} \ldots y^{\epsilon_{l}^{0}} r_{l}^{0} y w$ where $w$ is an alternating word representing $r_{m_{k}}^{k}$. Then $\|x\| \geq\|w\|=\left\|r_{m_{k}}^{k}\right\|=\left\|\tilde{a}^{-1} g_{n}\right\| \geq d\left(g_{n}, A\right)$.

We denote by $l(x)$ the length of a reduced presentation of $x \in G^{\prime}$. Let $P_{l}=$ $\{x \in G \mid l(x)=l\}$.

Proposition 7 Suppose that asdim $\leq \leq n, n>0$. Then asdim $P_{l} \leq n$ for all $l$.

Proof We use induction on $l$. We note that $P_{0}=G$ and $P_{l} \subset P_{l-1} y G \cup$ $P_{l-1} y^{-1} G$. We show first that asdim $\left(P_{l} \cap P_{l-1} y G\right) \leq n$. Let $r$ be given. We define $Y_{r}=P_{l-1} y N_{r}(A)$ where $N_{r}(A)$ is the $r$-neighborhood of $A$ in $G$. We check that $Y_{r} \subset N_{r+1}\left(P_{l-1}\right)$. Let $z \in Y_{r}$, then $z=x y g=x y a a^{-1} g=x \phi(a) y a^{-1} g$ where $x \in P_{l-1}, g \in N_{r}(A)$ and $a \in A$ with $\left\|a^{-1} g\right\| \leq r$. Then $x \phi(a) \in P_{l-1}$ and $d(x \phi(a), z)=\left\|y a^{-1} g\right\| \leq\|y\|+\left\|a^{-1} g\right\|=r+1$. Since $Y_{k}$ is coarsely isomorphic to $P_{l-1}$, by the induction assumption we have asdim $Y_{k} \leq n$. We consider the family of sets $x y G$ with $x \in P_{l-1}$. If $x y G \neq x^{\prime} y G$, then $y^{-1} x^{-1} x^{\prime} y \notin G$. A reduction in this word can occur only in the middle. Therefore $x^{-1} x^{\prime} \notin \phi(A)$. Moreover the reduced presentation of $y^{-1} x^{-1} x^{\prime} y$ after these reductions in the middle will be of the form $y^{-1} r_{1} \ldots r_{s} y$. Then $d\left(x y G \backslash Y_{r}, x^{\prime} y G \backslash Y_{r}\right)=$ $d\left(x y g, x^{\prime} y g^{\prime}\right)=\left\|g^{-1} y^{-1} x^{-1} x^{\prime} y^{\prime} g^{\prime}\right\|$. Since $g^{-1} y^{-1} x^{-1} x^{\prime} y^{\prime} g^{\prime}$ is a reduced presentation, by Proposition $6\left\|g^{-1} y^{-1} x^{-1} x^{\prime} y^{\prime} g^{\prime}\right\| \geq d(g, A)>r$. So, all the conditions of Theorem 1 are satisfied and, hence $\operatorname{asdim} P_{l-1} y G \leq n$. Similarly one can show that $\operatorname{asdim}\left(P_{l} \cap P_{l-1} y^{-1} G\right) \leq n$. Then the inequality asdim $P_{l} \leq n$ follows from the Finite Union Theorem.

Proof of Theorem 7 We consider a graph $T$ with vertices the left cosets $x G$. A vertex $x G$ is joined by an edge with a vertex $x g y^{\epsilon} G, g \in G, \epsilon= \pm 1$ whenever both $x$ and $x g y^{\epsilon}$ are reduced presentations. Since $l(x)=l(x g)$ for all $g \in G$, we can define the length of a vertex $x G$ of the graph. Thus all edges in $T$ are given an orientation and every vertex is connected by a path with the vertex $G$. Since the length of vertices grows along the orientation, there are no oriented cycles in $T$. We also note that no vertex can be the end point of two different edges. All this implies that $T$ is a tree. The group $G^{\prime}$ acts on $T$ by multiplication from the left. We note that the $r$-stabilizer $W_{r}(G)$ is contained in $P_{r}$. Hence by Proposition $7 \operatorname{asdim} W_{r}(G) \leq n$. Then Theorem 2 implies that asdim $G^{\prime} \leq 2 n+1$.

Remark Both the amalgamated product and the HNN extension are the fundamental groups of the simplest graphs of the group [12]. We note that theorems of Sections 4-5 can be extended to the fundamental groups of general graph of groups, since all of them are acting on the trees with the $R$-stabilizers having an explicit description. 


\section{Davis' construction}

We recall that a rightangled Coxeter group W is a group given by the following presentation:

$$
W=\left\langle s \in S \mid s^{2}=1,\left(s s^{\prime}\right)^{2}=1,\left(s, s^{\prime}\right) \in E\right\rangle
$$

where $S$ is a finite set and $E \subset S \times S$. A barycentric subdivision $N$ of any finite polyhedron defines a rightangled Coxeter group by the rule: $S=N^{(0)}$ and $E=\left\{\left(s, s^{\prime}\right) \mid\left(s, s^{\prime}\right) \in N^{(1)}\right\}$. The complex $N$ is called the nerve of $W$ (see [1]). We recall that the group $W$ admits a proper cocompact action on the Davis complex $X$ which is formed as the union $X=\cup_{w \in W} w C$, where $C=\operatorname{cone}(N)$ is called the chamber. Note that the action of $W$ on the set of centers of the chambers (i.e. cone vertices) is transitive. The orbit space of this action is $C$, and all isotropy groups are finite. Note that the Davis complex $X$ is contractible. There is a finite index subgroup $W^{\prime}$ in $W$ for which the complex $X / W^{\prime}$ is a classifying space. We denote $\partial C=N$. Let $X^{\partial}$ denote a subcomplex $X=\cup_{w \in W} w \partial C \subset X$. In [1] it was shown that there is a linear order on $W, e \leq w_{1} \leq w_{2} \leq w_{3} \leq \ldots$ such that the union $X_{n+1}^{\partial}=\cup_{i=1}^{n+1} w_{i} \partial C$ is obtained by attaching $w_{n+1} \partial C$ to $X_{n}^{\partial}$ along a contractible subset. Assume that $N \subset M$ is a subset of an aspherical complex $M$. We can build the space $X^{M}$ with an action of the group $W$ on it by attaching a copy of $M$ to each $w \partial C$. Then by induction one can show that every complex $X_{n}^{M}$ is aspherical and therefore $X^{M}$ is aspherical.

For every group $\pi$ with $K=K(\pi, 1)$ a finite complex, M. Davis considered the following manifold. Let $M$ be a regular neighborhood of $K \subset \mathbf{R}^{k}$ in some Euclidean space and let $N$ be a barycentric subdivision of a triangulation of the boundary of $M$. Then Davis' manifold is the orbit space $X^{M} / W^{\prime}$. It is aspherical, since $X^{M}$ is aspherical. We refer to the fundamental group $\Gamma=\pi_{1}\left(X^{M} / W^{\prime}\right)$ as Davis' extension of the group $\pi$. By taking a sufficiently large $k$, we may assume that the inclusion $N \subset M$ induces an isomorphism of the fundamental groups. Then in the above notation $\Gamma=\pi_{1}\left(X^{\partial} / W^{\prime}\right)$.

Theorem 8 If $\operatorname{asdim} \pi<\infty$, then $\operatorname{asdim} \Gamma<\infty$.

Proof Since $X^{\partial}$ is path connected, the inclusion $X^{\partial} \subset X$ induces an epimorphism $\phi: \Gamma=\pi_{1}\left(X^{\partial} / W^{\prime}\right) \rightarrow \pi_{1}\left(X / W^{\prime}\right)=W^{\prime}$. Let $K$ be the kernel. We note that $K=\pi_{1}\left(X^{M}\right)=\pi_{1}\left(X^{\partial}\right)=\lim _{\rightarrow}\left\{*_{i} \pi_{1}\left(w_{i} \partial C\right)\right\}$. It was proven in [7] that asdim $W<\infty$. The following lemma and Theorem 3 complete the proof.

Lemma 1 Assume that $K \subset \Gamma$ is supplied with the induced metric from $\Gamma$. Then $\operatorname{asdim} K \leq \operatorname{asdim} \pi$. 
Proof We fix a finite generating set $S$ for $\Gamma$. We consider $A_{w}=\pi_{1}(w \partial C)$, $w \in W$ as a subgroup of $K$ defined by a fixed path $I_{w}$ joining $x_{0}$ with $w\left(x_{0}\right)$. Assume that $A_{w}$ is supplied with the norm induced from $\Gamma$. We show that the inequality $\operatorname{asdim} A_{w} \leq$ asdim $\pi$ holds uniformly and by Theorem 4 we obtain that $\operatorname{asdim}\left(*_{w} A_{w},\|\|\right) \leq \operatorname{asdim} \pi$ for the norm \|\| generated by the norms on $A_{w}$. Then we complete the proof applying Proposition 5 .

Let $p: X^{\partial} \rightarrow \partial C$ be projection onto the orbit space under the action of $W$. Then $p=q \circ p^{\prime}$ where $p^{\prime}: X^{\partial} \rightarrow X^{\partial} / W^{\prime}$ is a covering map. We consider the norm on $\pi=\pi_{1}(\partial C)$ defined by the generating set $q_{*}(S)$. This turns $\pi$ into a metric space of bounded geometry. Then the homomorphism $q_{*}$ : $\pi_{1}\left(X^{\partial}\right)=\Gamma \rightarrow \pi_{1}(\partial C)=\pi$ is 1-Lipschitz map. The restriction of $q_{*}$ onto $A_{w}$ defines an isomorphism acting by conjugation with an element generated by the loop $p\left(I_{w}\right)$. Then according to Proposition 1 we have the inequality $\operatorname{asdim} A_{w} \leq \operatorname{asdim} \pi$ uniformly.

\section{References}

[1] M. Davis, Groups generated by reflections and aspherical manifolds not covered by Euclidean space, Ann. Math., (2) 117, (1983), 293-325.

[2] M. Davis, Coxeter groups and aspherical manifolds, Lecture Notes in Math. 1051 (1984), 197-221.

[3] M. Davis and J.-C. Hausmann, Aspherical manifolds without smooth or PL structure, Lecture Notes in Math., (2) 1370 (1989), 135-142.

[4] A. Dranishnikov, Asymptotic topology Russian Mathematical Surveys, 55 (6), (2000).

[5] A. Dranishnikov, On large scale properties of manifolds, Preprint, 1999. arxiv:math.GT/9912062

[6] A. Dranishnikov, Hypereuclidean manifolds and the Novikov Conjecture, Preprint of MPI, Bonn, 2000.

[7] A. Dranishnikov and T. Januszkiewicz, Every Coxeter group acts amenably on a compact space, Preprint, to appear in Topology Proceedings. arxiv:math.GT/9911245

[8] M. Gromov, Asymptotic invariants of infinite groups, Cambridge University Press, Geometric Group Theory, vol 21993.

[9] M. Gromov, Spaces and questions, Preprint, 1999.

[10] G. Mess, Examples of Poincare duality groups, Proc. Amer. Math. Soc. 110, no 4, (1990), 1145-1146.

[11] J. Roe, Coarse cohomology and index theory for complete Riemannian manifolds, Memoirs Amer. Math. Soc. No. 497, 1993. 
[12] J.-P. Serre, Trees, Springer-Verlag, 1980.

[13] G. Yu, The Novikov conjecture for groups with finite asymptotic dimension, Ann. of Math., 147, no 2, (1998), 325-355.

University of Florida, Department of Mathematics,

PO Box 118105, 358 Little Hall,

Gainesville, FL 32611-8105, USA

Email: dranish@math.ufl.edu, bell@math.ufl.edu

Received: 11 December 2000 Revised: 12 January 2001 